\title{
Oncogene-induced senescence and tumour control in complex biological systems
}

\author{
Lorenzo Galluzzi ${ }^{1,2,3} \cdot$ Ilio Vitale $e^{4,5,6}$
}

Received: 6 March 2018 / Accepted: 9 March 2018 / Published online: 17 April 2018

(c) ADMC Associazione Differenziamento e Morte Cellulare 2018

Mammalian cells experiencing potentially oncogenic conditions can undergo regulated cell death (RCD) [1] as well as an irreversible proliferative arrest known as cellular senescence [2]. Senescent cells are unable to proliferate owing to the permanent upregulation of cell cycle inhibitors [2], and are preferential targets for elimination by the immune system [3]. Thus, the net effect of senescence at the cell-intrinsic level is robust oncosuppression. However, senescent cells also release multiple cytokines that may support the proliferation of non-senescent neighbouring cells via paracrine mechanisms, de facto fostering tumour progression [4]. It is paramount to keep the cell-intrinsic and cell-extrinsic effects of cellular senescence well differentiated, especially in the context of complex systems in which cellular responses to damage can exhibit considerable degree of heterogeneity.

Sulli et al. [5] report that targeting two distinct nuclear hormone receptors that regulate circadian rhythmicity, namely, REV-ERB $\alpha$ (also known as NR1D1) and REV-ERB $\beta$ (also known as NR1D2), with pharmacological agonists is specifically lethal to malignant cells and cells that underwent oncogene-induced senescence (OIS). In particular, the authors of ref. [5] provide robust data linking the selectivity of REV-ERB agonists with the inhibition of

Lorenzo Galluzzi

deadoc@vodafone.it

1 Department of Radiation Oncology, Weill Cornell Medical College, 10065 New York, NY, USA

2 Sandra and Edward Meyer Cancer Center, 10065 New York, NY, USA

3 Université Paris Descartes/Paris V, 75006 Paris, France

4 Department of Biology, University of Rome "Tor Vergata", Rome, Italy

5 Regina Elena National Cancer Institute, Rome, Italy

6 Institute of General Pathology, Catholic University "Sacro Cuore", Rome, Italy autophagy, an evolutionarily old mechanism of adaptation that is particularly important for the metabolic reprogramming and survival of senescent cells [6], in a model of HRAS $^{\mathrm{G} 12 \mathrm{~V}}$-driven OIS. In the experimental setting employed by Sulli and colleagues [5], however, HRAS ${ }^{\mathrm{G} 12 \mathrm{~V}}$ overexpression results in approximately $60 \%$ of cells staining positively for senescence-associated $\beta$ galactosidase (as they report in Extended Data Fig. 9), implying that approximately $40 \%$ cells retained proliferative potential. Such $\beta$ galactosidase-negative cells (rather than their senescent, $\beta$ galactosidase-positive counterparts) are most likely responsible for the proliferation the authors observe over a period of 6 days (as they report in Fig. 3a, f), which can be robustly inhibited by pharmacological REV-ERB agonists. There is an abundant literature demonstrating that OIS is associated with an irreversible proliferative arrest and hence prevents, from a merely cell-intrinsic perspective, oncogenesis and tumour progression [7-11]. Sulli and collaborators [5] themselves lend additional support to their conclusions in an experimental model in which malignant progression is blocked by OIS, namely NRAS-driven naevi [12]. Nonetheless, in Fig. 4k, the authors of ref. [5] provide a visual summary of their findings that misleadingly depicts OIS as an intermediate step between normal cells and their fully malignant counterparts.

Our observations do not intend to detract from the findings of Sulli et al. [5]. On the contrary, we are fully convinced that their work may foster the development of new senolytic agents (i.e., molecules that are selectively cytotoxic for senescent cells) based on REV-ERB agonism, which is particularly relevant given the major role played by senescent cells in a variety of pathologies linked to organismal aging [13, 14]. Rather, the considerations above intend to raise awareness on two points with profound pathophysiological and therapeutic implications (Fig. 1). First, complex systems including cancer cell lines growing in vitro respond to all stimuli with a degree of heterogeneity that is often underestimated. The percentage of cells undergoing OIS upon $\mathrm{HRAS}^{\mathrm{G} 12 \mathrm{~V}}$ overexpression in the 


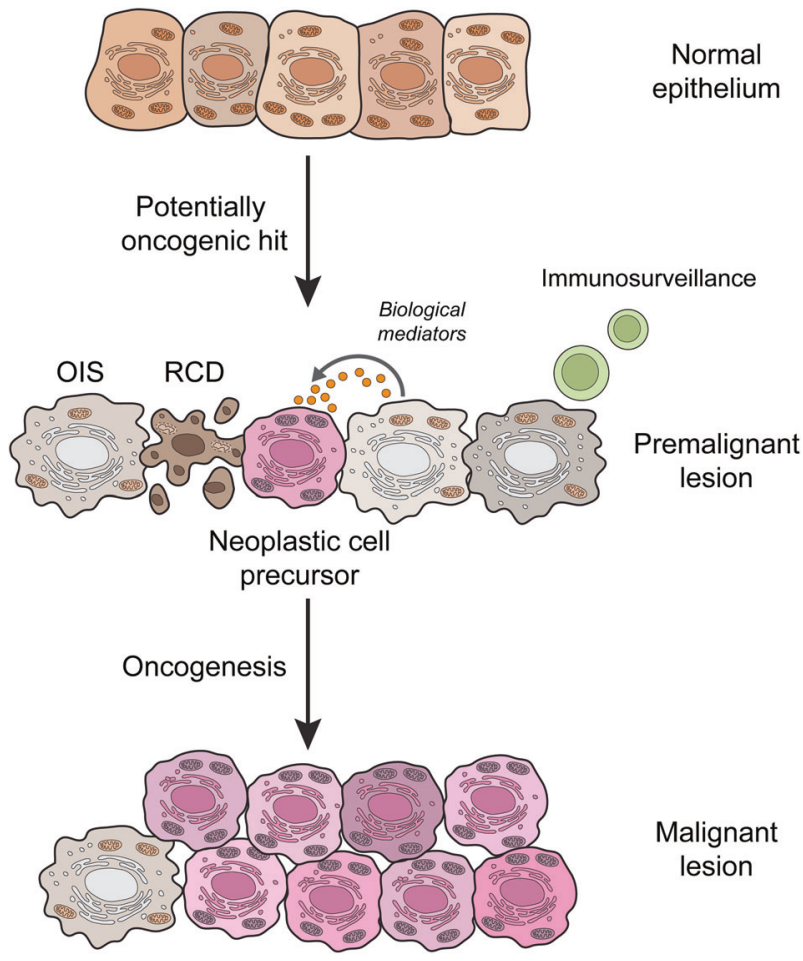

Fig. 1 Oncogene-induced senescence and tumour control in complex biological systems. Heterogeneous biological systems including cell lines growing in vitro as well as cells constituting animal tissues can respond to potentially tumorigenic stimuli, such as the activation of one oncogene, by undergoing regulated cell death (RCD) or oncogeneinduced senescence (OIS). At the cell-intrinsic level, these mechanisms mediate robust oncosuppressive effects as they eliminate neoplastic cell precursors (RCD), or permanently abrogate their proliferative potential (OIS). Moreover, cells undergoing OIS are preferentially recognised and eliminated by the immune system. However, senescent cells that survive such an immune control secrete a variety of biological factors that stimulate the proliferation of neighbouring, non-senescent cells, de facto fostering tumour progression by cell-extrinsic mechanisms. This scenario provides a good example of the heterogeneity of responses in complex biological systems as well as of the co-existence of cell-intrinsic and -extrinsic mechanisms emanating from the same cue but not necessarily culminating in the same outcome

experimental setting employed by Sulli and collaborators [5] provides a nice example of such a functional heterogeneity. Second, multiple biological processes operate both at intracellular (cell-intrinsic) and microenvironmental or systemic (cell-extrinsic) levels, and the net outcome of these pathways does not always match. For instance, while OIS mediates purely oncosuppressive effects at the cell-intrinsic level as it prevents the proliferation of potentially malignant cells [2] and promotes their elimination by the immune system [3], multiple biological mediators secreted by senescent cells, including components of the so-called senescence-associated secretory phenotype may promote tumour progression at the cell-extrinsic level [4]. We are convinced that keeping these two points under attentive consideration may help researchers from a variety of biological disciplines with interpreting their findings in the best possible way, de facto fostering the progress of science.

Author Contributions LG conceived the article and wrote the first version of the manuscript. IV provided critical input and prepared the figure. All authors approved the article in its final form.

\section{Compliance with ethical standards}

Conflict of interest The authors declare that they have no conflict of interest.

\section{References}

1. Galluzzi L, et al. Immunogenic cell death in cancer and infectious disease. Nat Rev Immunol. 2017;17:97-111.

2. Munoz-Espin D, et al. Cellular senescence: from physiology to pathology. Nat Rev Mol Cell Biol. 2014;15:482-96.

3. Kang TW, et al. Senescence surveillance of pre-malignant hepatocytes limits liver cancer development. Nature. 2011;479:547-51.

4. Demaria M, et al. Cellular senescence promotes adverse effects of chemotherapy and cancer relapse. Cancer Discov. 2017;7:165-76.

5. Sulli G, et al. Pharmacological activation of REV-ERBs is lethal in cancer and oncogene-induced senescence. Nature. 2018;553:351-5.

6. Galluzzi L, et al. Molecular definitions of autophagy and related processes. EMBO J. 2017;36:1811-36.

7. Braig $\mathrm{M}$, et al. Oncogene-induced senescence as an initial barrier in lymphoma development. Nature. 2005;436:660-5.

8. Chen Z, et al. Crucial role of p53-dependent cellular senescence in suppression of Pten-deficient tumorigenesis. Nature. 2005;436:725-30.

9. Collado M, et al. Tumour biology: senescence in premalignant tumours. Nature. 2005;436:642.

10. Bartkova $\mathrm{J}$, et al. Oncogene-induced senescence is part of the tumorigenesis barrier imposed by DNA damage checkpoints. Nature. 2006;444:633-7.

11. Di Micco R, et al. Oncogene-induced senescence is a DNA damage response triggered by DNA hyper-replication. Nature. 2006;444:638-42.

12. Michaloglou C, et al. BRAFE600-associated senescence-like cell cycle arrest of human naevi. Nature. 2005;436:720-4.

13. Baker DJ, et al. Clearance of p16Ink4a-positive senescent cells delays ageing-associated disorders. Nature. 2011;479:232-6.

14. Baker DJ, et al. Naturally occurring p16(Ink4a)-positive cells shorten healthy lifespan. Nature. 2016;530:184-9. 